\title{
CLINICAL STUDY OF MYCOTIC KERATITIS HUBLI, IN NORTH KARNATAKA.
}

Jyothi N. S1, Manish. K², Sowmya K. V³.

1. Assistant Professor, Department of Ophthalmology, ESIC Medical College \& Hospital, Gulbarga, Karnataka.

2. Assistant Professor, Department of Forensic Medicine \& Toxicology, ESIC Medical College \& Hospital, Gulbarga, Karnataka.

3. Consultant Ophthalmologist, Yallapur, Karnataka.

\section{CORRESPONDING AUTHOR:}

Dr. Jyothi N. S,

Assistant Professor,

Dept of Ophthalmology, ESICH, Gulbarga, Karnataka.

E-mail: jyothi2406@rediffmail.com

\begin{abstract}
PURPOSE: To determine, the epidemiological characteristics and risk factors predisposing to fungal keratitis in Hubli, south India. To know the importance of KOH wet mount for rapid detection of fungal keratitis for institution of antifungal therapy as earliest possible. To know the statistical significance regarding the common type of fungi that involves the cornea and prognosis with regard to the available mode of therapy and their results. METHOD: In one year period, out of 112 patients presented with corneal ulcer, 48 (42.85\%) were clinically suspected as having mycotic keratitis. Sensitivity of $\mathrm{KOH}$ wet mount preparation in our study is $96.25 \%$ in our study. Fusarium (53.84\%) was most commonly isolated fungus. In our study all 48 cases were treated with Monotherapy, in that 28 cases patients deep stromal involvement were treated with combined therapy. About 15 patients received surgical treatment. 17(35.41\%) cases had only HM positive and one case (2.08\%) with only perception of light. and 2(6.25\%) cases with no perception and rest had fairly good vision. CONCLUSION: These findings have important public health implications for the early diagnosis in suspected cases of mycotic keratitis and institution of specific therapy at earliest to avoid hazardous effects on cornea.
\end{abstract}

INTRODUCTION: corneal blindness accounts for 20-30\% of all blindness in developing countries of the world. Suppurative keratitis is the leading cause with mycotic keratitis constituting half of the cases in north Karnataka. Morbidity in mycotic infection tends to be higher compared to bacterial keratitis because diagnosis is often delayed due to negligence towards ocular trauma, inappropriate treatment and wide spread use of topical antibiotics and steroids for any case of corneal ulcer.

KIMS Hubli, being tertiary care hospital and major referral center, we get more than two three cases of mycotic keratitis per OPD. Considering its prevalence it is thought worthwhile to take up clinical study of mycotic keratitis to diagnose the case on suspicion with laboratory investigation and to start antimycotic treatment early for better results.

\section{AIMS AND OBJECTIVES:}

Clinical study of mycotic keratitis concerns with:

1. To know the incidence of mycotic keratitis. 


\section{ORIGINAL ARTICLE}

2. To study $\mathrm{KOH}$ sensitivity in diagnosing fungal corneal ulcers in comparison with fungal growth on culture.

3. The statistical significance regarding the common type of fungi that involve the cornea.

4. The prognosis with regard to the available mode of therapy and their results.

METHODOLOGY: The clinical study of mycotic keratitis was conducted in the department of Ophthalmology, KIMS, Hubli from 1st September 2007 - 31st August 2008.

Inclusion criteria: Corneal ulceration was defined as 'loss of corneal epithelium with underlying stromal infiltrate and suppuration associated with signs of inflammation with or without hypopyon'. Clinical features such as longer duration, elevated, plaque like serrated/feathery margins of the ulcer, raised slough, dry surface (texture),satellite lesions, Weiss ring, endothelial plaque, coloration of the ulcer other than yellow (suggestive of diatomaceous fungi) and thick hypopyon were noted and categorized as patients with fungal keratitis.

Exclusion criteria: Non suppurative keratitis including viral keratitis, neurotrophic ulcers, degenerative causes were excluded as were Moorens ulcer, interstitial keratitis, sterile neurotrophic ulcers and any ulcer associated with systemic autoimmune conditions.

Clinical procedure: Visual acuity was recorded in standard manner. All patients were subjected to a detailed slit lamp biomicroscopic examination. Size of ulcer was roughly recorded with fluorescent stain and Rose Bengal stain under local anesthesia. Depth of the lesion, stromal infiltrate, ulcer margin, thinning, floor, satellite lesions, vascularity, pigmentation, any impacted foreign body and hypopyon were noted.

Material was collected from conjunctiva, sac area, corneal ulcer scrapings. Materials used by patients (eye drop bottles, lens solutions), and corneal button after therapeutic keratoplasty were collected. The collected material was submitted for Gram staining, Giemsa staining and $\mathrm{KOH}$ wet mount preparation. Fungal elements are observed under microscope. Rest of the material was sent for culture .Commonly used medias were, Blood agar plate(bacteria,35-37 deg\& fungi, room temp), Chocolate agar plate (aerobes and facultative anaerobes,35 deg), SDA without cycloheximide, SDA with cycloheximide(fungus), Thioglycolate broth(anaerobes,35-37 deg).

Remaining scrapings were utilized for histopathology

Interpretation of Fungal results: $10 \% \mathrm{KOH}$ wet mount is useful in detecting for hyphal elements, budding cells and fungal morphologies. Lab criteria for culture positive include (1)growth on 2 or more media (2)Growth on at least one medium of the same organism identified in smears (3)Confluent growth at the inoculation site on at least one solid medium or repeat isolation from same patient. These criteria are more applicable to bacteria and fungi.

TREATMENT: The standard treatment protocol was followed based on $\mathrm{KOH}$ staining categorizing causative fungal element as filamentous and non filamentous.

Epithelium was debrided every 24-48 hrs to increase drug penetration \& to reduce fungal load along with debris. One drop of Natamycin suspension $5 \%$ was used every hour during day time and every 2 hours at night time used for filamentous fungi. Dosing was tapered according to the clinical response Nystatin ointment was supplemented in nonresponsive cases. Oral Fluconazole $150 \mathrm{mg}$ OD added when ulcer was more than $6 \mathrm{~mm}$ in diameter, deeper than the anterior one half of the stroma or if anterior chamber exudates were present. 


\section{ORIGINAL ARTICLE}

Antifungal treatment was supplemented with Broad spectrum antibiotics, Cycloplegic, Antiinflammatory agents, Vitamin $\mathrm{C}$ and Lubricating eye drops. For mycotic keratitis with secondary glaucoma anti-glaucoma medications were prescribed. For corneal ulcer with small perforation Bandage contact lens, Cyanoacrylate glue and conjunctival hooding was done. For larger perforations, therapeutic keratoplasty was done.

Follow up: Patients were followed weekly for one month then every 15 days up to 6 months. Thorough Examination was done at each visit. Treatment was decided upon further based on clinical response. Outcome measures: Symptomatic improvement, comfort of patient, remission of signs, reduction in size of ulcer and improvement in visual acuity.

OBSERVATIONS AND RESULTS: The clinical study of mycotic keratitis, conducted in the department of ophthalmology, KIMS, Hubli from $1^{\text {st }}$ September 2007 to $1^{\text {st }}$ august 2008.

During this period, the following observations were made. Out of 112 patients with suppurative keratitis, 48 (42.85\%) were clinically suspected of mycotic keratitis and positive for fungal elements (Table: I). The higher incidence of males (Table: II) is probably due to their involvement in outdoor activities which predispose them to trauma with vegetable matter and other types of traumas with causative agent contaminated with fungus (Table: IV).

Maximum cases seen in the age group of 41-60 (31.25\%) years, Most of them were agriculturists (Table: III). A significant higher incidence of mycotic keratitis was observed during the harvesting period and dry windy season i,e June-august(33.33\%) \&September to November(52.08\%).

In the present study, $77.08 \%$ of mycotic keratitis was seen in low socio economic status. In the present study $95.83 \%$ cases had used drugs prior to consultation. Peripheral fungal keratitis accounted to $27.09 \%$.Diffuse fungal keratitis was seen in $33.33 \%$ of cases. In the present study of mycotic keratitis size of the fungal keratitis varied from $1 \mathrm{~mm}$ to the total corneal involvement.

Corneal ulcer with perforation was seen in $17(35.44 \%)$ patients. $10(20.84 \%)$ patients developed secondary glaucoma. Panophthalmitis was seen in one (2.08\%) 12 (25.08\%)cases had visual acuity of $6 / 18$ to $6 / 6 / 60,25(52.08 \%)$ cases had vision of counting fingers' $1-5 \mathrm{mts} \& 7$ (14.56\%) with perception of hand movements.3 (6.20\%) patients had perception of light and one (2.08\%)with no perception on presentation.

Sensitivity of $\mathrm{KOH}$ wet mount preparation in our study is $96.25 \%$

Fusarium was the commonest fungal species (Table: VIII). In the present study mixed infections were seen in $20(17.85 \%)$.In that infection with Staph aureus was seen in $12(60 \%)$ cases, Streptococci in 5 (25\%) cases, Enterobacter in 1 (5\%) case and Pseudomonas in 2(10\%) cases.

In the present study of mycotic keratitis, all 48 were patients treated with medical line of treatment i.e. Natamycin 5\% sups hrly. 28 (58.33\%) cases of deep stromal fungal corneal ulcer with or without hypopyon (Table: IX) were treated with Natamycin 5\% suspension hrly along with systemic, Tab. Fluconazole 200-600mg/day QID for 2-3 weeks.

In all 48 cases, treatment was started with debridement of the ulcer (Table: X). Conjunctival hooding was done in 14.62\%) cases with small perforation. Bandage contact lenses were prescribed in 9 cases. Dacryocystectomy was done in 6 cases Therapeutic keratoplasty \&evisceration was done in single cases. In the present study of mycotic keratitis, 3 (6.25\%) cases had fairly good vision i.e 6/24-6/36, 10(17.83\%) had 6/36 to 6/60, 15(31.25\%) patients had CF 1 - 


\section{ORIGINAL ARTICLE}

5mts.and $17(35.41 \%)$ cases had only HM positive. One case $(2.08 \%)$ was left with only perception of light. and 2(6.25\%) cases were with no perception of light (Table: XI).

DISCUSSION: In the clinical study of mycotic keratitis, out of 112 cases suppurative keratitis $48(42.85 \%),[2,4,7,9,15]$, cases were mycotic keratitis on clinical suspicion and fungal staining, as most of our patients were agriculturists and trauma with organic matter is the common predisposer, increasing likelihood of fungal corneal ulcer.

The higher incidence in male with age group 21-60 due to outdoor activities which predispose them to trauma with vegetable matter and other types of traumas with causative agent contaminated with fungus. Most of the patients were farmers $(43.74 \%)[8,14]$, labourers $(31.25 \%)$, and gardeners $(12.50 \%)$ as they are exposed to injury more often. The higher incidence of mycotic keratitis in rural area (75\%) may be due to their exposure to agricultural work, lack of education, illiteracy, and poverty.

History of injury with Vegetable mater, ${ }^{[12,14]}$ accounted for 17 cases $(35.41 \%) .0$ ther modes of injury were trauma with Nail in 12 cases (25.00\%), and Stone in 10 cases (20.83\%),[5,12,14].A significant increase in incidence was observed during the harvest period and dry windy season likely to be a direct consequence of increased agricultural activity before and immediately following rains. Associated factors, ${ }^{[3]}$ like Diabetes mellitus, chronic dacryocystitis, Malnutrition, Alcoholism and Immuno compromised status were seen. $95.83 \%$ cases in the present study had used drugs prior to consultation (Table: VI).

The incidence of central mycotic keratitis was accounted to 39.58\%.Peripheral fungal keratitis accounted to $27.09 \%$. Diffuse fungal keratitis with poor prognosis was seen in $33.33 \%$ of cases.. Size of the fungal keratitis varied from $1 \mathrm{~mm}$ to the total corneal involvement. The prognosis of ulcers, which have size of more than $5 \mathrm{~mm}$ was not good due to corneal scarring .On presentation $20(41.66 \%)$ of cases had superficial stromal involvement \& $28(58.34 \%)$ deep stromal involvement $.55 .33 \%$ of cases had hypopyon. $85.41 \%$ of cases had feathery margins, $75.00 \%$ of cases had dry surface, $47.91 \%$ cases had Elevated surface $79.16 \%$ cases had Satellite lesions. Whitish round lesion with surrounding infiltration was seen in $2.08 \%$ cases (Table: VII).

In our study visual acuity of $6 / 18$ to $6 / 6 / 60(25.08 \%)$, counting fingers $1-5 \mathrm{mts}(52.08 \%) \&$ $7(14.58 \%)$ with perception of hand movements.3 (6.25\%) patients had perception of light and one $(2.08 \%)$ with no perception. Complicated mycotic keratitis (Table: IX), was seen with perforation (35.44\%), secondary glaucoma (20.84\%) and Panophthalmitis (2.08\%). Sensitivity of KOH wet mount preparation in our study is $96.25 \%,[1,3]$ that shows $\mathrm{KOH}$ wet mount preparation as a reliable criterion for the diagnosis of fungal keratitis. Fusarium, ${ }^{[2,3]}$ formed the common fungal isolate in our study.

All 48 cases treatment was started with debridement of ulcer, in that $68.76 \%$ were treated with medical line of treatment and $31.24 \%$ with surgical line of treatment, [7, 10]. Out of 48 cases, $17(35.41 \%)$ cases had only HM positive. one case $(2.08 \%)$ with only perception of light. and $2(6.25 \%)$ cases with no perception and rest had good visual prognosis. Visual prognosis at the end of follow up was poor as corneal scaring was the predominant outcome in most of the cases. The visual outcome in our study is almost similar to the study by Naseem et al, [11] and worst than Lt Col SS Mann, [10]. As most of our patients presented after 1 week of onset of symptoms, treatment was delayed and ulcer was progressed on presentation. 
CONCLUSION: India being an agricultural country, the incidence of mycotic keratitis is significantly high. Mycotic keratitis has tendency to progress slowly with minimal symptoms hence the patients are provoked to apply local native medicines and approach the doctor very late. Lack of laboratory identification especially in peripheral hospital adds to the problem.

This study signifies the importance of keeping in mind fungus as one of the inflicting agent in causation of corneal ulcer especially when predisposing factors like corneal trauma with organic matter, Chronic dacryocystitis, Diabetes Mellitus etc are present and also by looking at the clinical features and taking help of rapid diagnostic tests like Gram's stain, KOH stain, one can predict the diagnosis early. With timely specific treatment, one can combat the disease efficiently and thus prevent the eye going in for blindness

\section{BIBLIOGRAPHY:}

1. "Trends in contact lens-associated microbial keratitis in Southern India" Ophthalmology, Savitri Sharma, Sujatha Gopalakrishnan, Murali K Aasuri, Prashant Garg, Gullapalli N Rao. Ophthalmology 2003; 110; 1: 138-143.

2. "Aetiology of suppurative corneal ulcers in Ghana and south India, and epidemiology of fungal keratitis". A K Leck, P A Thomas, M Hagan, J Kaliamurthy, E Ackuaku, M John, M J Newman, F S Codjoe, J A Opintan, C M Kalavathy, V Essuman, C A N Jesudasan, G J Johnson.Br J Ophthalmol. 2002; 86,1211-1215.

3. "The Epidemiological Features and Laboratory Results of Fungal Keratitis-A 10-Year Review at a Referral Eye Care Center in South India". Usha Gopinathan, Prashant Garg, Merle Fernandes, Savitri Sharma, Sreedharan Athmanathan, and Gullapalli N. Rao. Cornea 21: $555-559,2002$

4. "Epidemiological characteristics and laboratory diagnosis of fungal keratitis - A three-year study". Bharathi MJ, Ramakrishnan R, Vasu S, Meenakshi R, Palaniappan R. Indian J Ophthalmol 2003;51,315-21

5. "Study of mycotic keratitis". Poria VC, Bharad VR, Dongre DS, Kulkarni MV. Indian J Ophthalmol1985;33,229-31

6. "The diagnosis and management of keratomycosis". Forster RK, Rebell G Arch Ophthalmol 1975; 93,975-78.

7. Smolin and Thoft's The Cornea Scientific Foundations and Clinical PracticeAuthor(s): C. S Foster MD, FACS Dimitri T Azar MD Claes H Dohlman MD Edition: Fourth .Pg no.405-425.

8. "Study of fungal Keratitis". Dutta LC, Dutta D, Mohanty P, Sharma J. Indian J Ophthalmol 1981; 29,407-40.

9. "The correlation between environmental and ocular fungi". Sandhu DK, Randhawa IS, Singh D. Indian J Ophthalmol 1981; 29,177-82.

10. "Medical and Surgical Management of Keratomycosis". Lt Col SS Mann, Col (Retd) J Singh, VSM, Col D Kalra, VSM, Col JKS Parihar, VSM, SM, N Gupta, Lt Col P Kumar, MJAFI 2008.

11. "Fungal keratitis: A two years retrospective study". Naseem A, Nawaz A, Jan S, Muhammad S. Pak J Ophthalmology 2001; 17: 129-33.

12. "Mycotic infections of cornea". Prasad S, Nema HV. Indian J Ophthalmol 1982;30,81-5. 
13. "Spectrum of Fungal Keratitis in North India". Chowdhary, Anuradha MD*; Singh, Kirti MD, DNB, FRCS† Cornea: January 2005; 24; 1, 8-15.

14. "Epidemiology and aetiological diagnosis of corneal ulceration in Madurai, south India". M Srinivasan, Christine A Gonzales, Celine George, Vicky Cevallos, Jeena M Mascarenhas, B Asokan, John Wilkins, et al .British Journal of Ophthalmology 1997;81,965-971.

* Table: 1 : incidence of keratomycosis

\begin{tabular}{|c|c|c|}
\hline Total number patients with suppurative keratitis & $\begin{array}{c}\text { Clinically suspected } \\
\text { mycotic keratitis }\end{array}$ & Percentage \\
\hline 112 & 48 & $42.85 \%$ \\
\hline
\end{tabular}

Table: II: Sex distribution

\begin{tabular}{|l|c|c|}
\hline \multirow{2}{*}{ Sex } & \multicolumn{2}{|c|}{ Fungal corneal ulcer } \\
\cline { 2 - 3 } Mumber & Percentage \\
\hline Males & 31 & $64.58 \%$ \\
\hline Females & 17 & $35.42 \%$ \\
\hline
\end{tabular}

Table: III: occupation

\begin{tabular}{|c|c|c|}
\hline \multirow{2}{*}{ Occupation } & \multicolumn{2}{|c|}{ Fungal corneal ulcer } \\
\cline { 2 - 3 } & Number & Percentage \\
\hline Agriculturists & 21 & $43.75 \%$ \\
\hline Labourers & 15 & $31.25 \%$ \\
\hline Gardeners & 06 & $12.50 \%$ \\
\hline Housewife & 03 & $6.25 \%$ \\
\hline Student & 02 & $4.16 \%$ \\
\hline Businessmen & 01 & $2.08 \%$ \\
\hline
\end{tabular}

Table: IV: Modes of trauma

\begin{tabular}{|l|c|c|}
\hline \multirow{2}{*}{ Modes } & \multicolumn{2}{|c|}{ Fungal corneal ulcer } \\
\cline { 2 - 3 } & Number & Percentage \\
\hline Vegetable matter & 17 & $35.42 \%$ \\
\hline Stone & 10 & $20.83 \%$ \\
\hline Finger nail & 12 & $25.00 \%$ \\
\hline Nil & 09 & $18.75 \%$ \\
\hline
\end{tabular}




\section{ORIGINAL ARTICLE}

Table: V: Associated factors

\begin{tabular}{|l|c|c|}
\hline \multirow{2}{*}{ Associated factors } & \multicolumn{2}{|c|}{ Fungal corneal ulcer } \\
\cline { 2 - 3 } & Number & Percentage \\
\hline Diabetes mellitus & 15 & $31.25 \%$ \\
\hline Chronic dacryocystitis & 4 & $8.32 \%$ \\
\hline Alcoholism & 06 & $12.50 \%$ \\
\hline Malnutrition & 08 & $16.66 \%$ \\
\hline Immuno compromised state & 01 & $2.10 \%$ \\
& & \\
\hline
\end{tabular}

Table: VI: Prior use of drugs

\begin{tabular}{|l|l|l|}
\hline \multirow{2}{*}{ Drugs } & \multicolumn{2}{|l|}{ Fungal corneal ulcer } \\
\cline { 2 - 3 } & Number & Percentage \\
\hline Antibiotics & 25 & $52.08 \%$ \\
\hline Antibiotics with steroids & 14 & $29.16 \%$ \\
\hline Local medicine & 07 & $14.58 \%$ \\
\hline Nil & 02 & $4.18 \%$ \\
\hline
\end{tabular}

TABLE: VII: Ulcer characteristics

\begin{tabular}{|l|l|l|}
\hline \multirow{2}{*}{ Clinical manifestation } & Fungal corneal ulcer \\
\cline { 2 - 3 } & Number & Percentage \\
\hline Feathery margins & 41 & $85.41 \%$ \\
\hline Dry surface & 36 & $75.00 \%$ \\
\hline Elevated surface & 23 & $47.91 \%$ \\
\hline Satellite lesions & 38 & $79.16 \%$ \\
\hline Hypopyon & 28 & $55.33 \%$ \\
\hline $\begin{array}{l}\text { Whitish round lesion with } \\
\text { surrounding infiltration }\end{array}$ & 01 & $2.08 \%$ \\
\hline
\end{tabular}

Table: VIII: Different species identified in culture positives

\begin{tabular}{|l|l|l|}
\hline \multirow{2}{*}{ Organism } & \multicolumn{2}{|l|}{ Fungal corneal ulcer } \\
\cline { 2 - 3 } & Number & Percentage \\
\hline Fusarium & 11 & $42.30 \%$ \\
\hline Aspergillus & 09 & $34.61 \%$ \\
\hline Pencillium & 02 & $7.69 \%$ \\
\hline Cephalosorium & 03 & $11.53 \%$ \\
\hline Candida & 01 & $3.87 \%$ \\
\hline
\end{tabular}




\section{ORIGINAL ARTICLE}

Table: IX: Fungal corneal ulcer with complications

\begin{tabular}{|l|l|l|}
\hline Complications & Number & Percentage \\
\hline Perforation & 17 & $35.44 \%$ \\
\hline Secondary glaucoma & 10 & $20.84 \%$ \\
\hline Panophthalmitis & 01 & $2.08 \%$ \\
\hline
\end{tabular}

Table: X: Surgical treatment

\begin{tabular}{|l|l|l|}
\hline & Number & Percentage \\
\hline Debridement & 48 & $100 \%$ \\
\hline Conjunctival hooding & 07 & $14.62 \%$ \\
\hline Bandage contact lens & 9 & $18.75 \%$ \\
\hline Dacryocystectomy & 6 & $12.54 \%$ \\
\hline Keratoplasty & 01 & $2.08 \%$ \\
\hline Evisceration & 01 & $2.08 \%$ \\
\hline
\end{tabular}

Table: XI: Visual outcome at last follow up

\begin{tabular}{|l|l|l|}
\hline Visual acuity & No. & Percentage \\
\hline $\mathbf{6 / 2 4 - 6 / 3 6}$ & 3 & $6.25 \%$ \\
\hline $\mathbf{6 / 3 6 - 6 / 6 0}$ & 10 & $17.83 \%$ \\
\hline CF 1-5mts & 15 & $31.25 \%$ \\
\hline HM+VE & 17 & $35.41 \%$ \\
\hline PL+VE & 1 & $2.08 \%$ \\
\hline PL -VE & 2 & $4.18 \%$ \\
\hline
\end{tabular}

